\title{
Modelling and Design Optimization of Coanda-Effect Application on Surface Particle Removal
}

\author{
Bagus Bhirawa Putra ${ }^{\mathrm{a}}$, Xu He ${ }^{\mathrm{b}}$, Huang Xinliang ${ }^{\mathrm{c}}$ \\ College of Mechanical and Electrical Engineering, Harbin \\ Engineering University, Harbin 150001, China \\ abagus@hrbeu.edu.cn, brailway_dragon@163.com, \\ c297266932@qq.com
}

\begin{abstract}
In this paper, decoupled form of CFD methods considering important design parameters is applied to model and predict the surface flow on fluids. Application of Coandaeffect on-going trends of particle removal methods for determination and prediction of cleaning performance in a camera lens is a new research area due to its significant role in design, evaluation and optimization. Numerical analysis of cleaning performance in term of particle drag force coefficient of nozzle and energy efficiency is carried out for surface radii, cover lengths and angle of attack. Furthermore, an optimization method for achieving optimal particle cleaning is presented. The optimization problem is formulated based on independent variables including lens surface radius $r$, camera cover length $l_{1}$ and $l_{2}$, and angle of attack $\theta$ with the objectives to maximize pressure flow and drag force coefficient while minimizing the dynamic turbulence flow. To establish this condition, a novel design of particle removal is introduced and simulation study is carried out in FLUENT to investigate the growth and the structure of surface disturbances on a flat and curved surface by a coaxial airflow in accordance with the pressure distribution and shear stress. And the genetic algorithm in I-SIGHT is used to obtain optimized particle removal for camera cover design. A method to apply the optimization results for optimum particle drag forces ability is discussed.
\end{abstract}

Keywords-Coanda-effect, Particle removal, Optimization, Particle drag force.

\section{INTRODUCTION}

The result of fluid behaviours in interaction with surfaces, other fluids or, indeed, with itself considered to be common interest of most fluid phenomena. While the standard approach to fluid dynamics can be said is effective in providing a means of calculating a wide range of fluid behaviour and seen as interaction with each other or with any given surface. These general classes of phenomena, which may be observed in both gaseous and liquid jets, are known as the Coanda-effect. An analytical and numerical solution that approximates a two and three-dimensional Coanda flow is proposed. Since between the tangential components of the momentum equation, with the validity of the results is being limited to approximate values. In order to determine its advantages and limitation, both external and internal flows of the FLUENT code are analysed which emphasising on the Coanda-effect. In the design of any complex system, it customary to find that the decomposition of such a system divides the analysis of the problem into several smaller sections that focus on designated application such as particle removal apparatus [1].

\section{BERNOULLI AND COANDA FLOW}

In order to obtain the particle velocities in the cell approximately equal in magnitude, the velocity should be kept small enough. The cause of the Coanda-effect, in some circumstances could refer to a flow that stirred up a pressure differential. Strictly speaking, because particles will move lateral to the flow after striking protuberances on the surface, Bernoulli's equation does not apply over a real free surface. According to Statistical Mechanics, the root mean square of the or particle speed, $v_{r m s}$ is not related to the pressure, $P$ but only to the temperature, $T$ and the particle mass, $m$.

$$
\frac{1}{2} m v_{r m s}^{2}=\frac{3}{2} k_{B} T
$$

Where $k_{B}$ is Boltzmann's constant, and thus no matter what $p_{0}$ is, $\left(\overline{\boldsymbol{v}}_{\boldsymbol{x}}\right)_{0}$ cannot exceed the particle speed, $v_{r m s}$, corresponding to the temperature $T$. It is very important to have a proper detailed design of this region, in order to achieve the best mixing and delivery of the flow as define, which ensure the pressure is applied. The phenomenon in which an air flow attaches to an adjacent wall which curves away from this flow, usually referred as the Coanda-effect. It is due to the decrease in the particle density $\rho$, in the boundary layer. Particles enter and leave just above the surface of the airfoil, in a given constant volume, $V$. An equation for pressure in an airfoil can be formed by letting $\eta(s)$ to be the particle density at $s$ on the top of the airfoil. Hence the particle density in Coanda flow is described as $\eta$.

$$
p=\eta k T
$$

\section{REMOVAL OF PARTICLES}

\section{A. Mechanical Method}

In this study, the discussion proposes the concept of particle removal on camera lens surfaces which are an integral part of a visual system in various applications, where cameras are standard equipment on most of today's activity. By understanding the present basic theory and 
design methodology, this would capture the outline for future developments of the novelty in particle removal methods. As shown in Fig. 1, each camera design has unique features different from one another; however there are some similarities in their functionality.

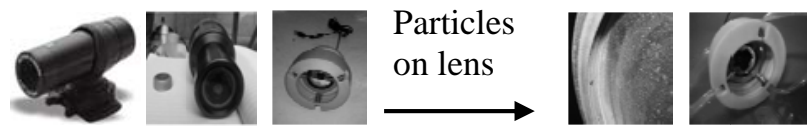

Figure 1. Typical case of particles concentration on camera lens surface [2]

\section{B Utilization of Jet Nozzle for Particle Removal}

Under the right performance of spraying, the charged particles will not be allowed to deposit, but will be encouraged to move along the surface pattern following the course of the sprayed media [3]. In the particle removal process, the fluid drag force is considered as the main mechanism [4] which is shown in Fig. 2. For most liquid physical assistance techniques which are applied either in parallel or typically to the cleaning substrate, this mechanism is entirely a dominant factor [5].The equation of drag force for based cleaning with different types of challenging materials [6] can be approached by the given formula as follows,

$$
F_{D}=C_{D}\left(\frac{\rho V_{f}^{2}}{2}\right) \times\left(\frac{\pi d_{p}^{2}}{4}\right)
$$

In this way, the surface will be kept clean of particle deposits. Anirban Guha et al. [7] have carried out some numerical and theoretical investigation work on air jet especially for cleaning processes. In the engineering field, high-speed air jets in various environments have been successfully applied [8,9] such as cutting, cleaning and surgery. In addition to jets' application, particle cleaning used in camera surfaces could be a fitted combination towards invention which is one step ahead in surface cleaning technology, yet the requirements are not necessarily the same as that of drilling and cutting in terms of nozzle velocity.
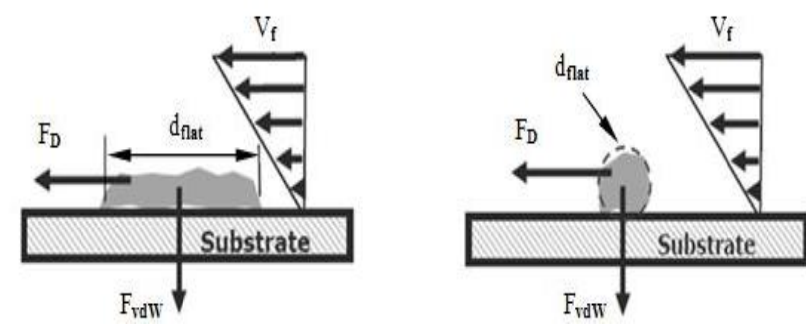

Figure 2. Attraction forces during particle removal process [6] for (left) Large flat particle area with characteristic diameter, $d_{f l a t}$, and (right) Spherically shaped particle with characteristic diameter, $d_{p}$

For further investigation on the pressure distribution on a target surface, like plate and glass positioned at a given axial distance from the outlet nozzle, Leach et al. [10] noted that the normalized pressure is actually independent along the radial direction when the distribution throughout the centreline of a jet is dependent on the nozzle geometry.

\section{NUMERICAL ANALYSIS OF LAMINAR AND TURBULENT COANDA FLOW}

The particle removal system is designed as an axisymmetric device that uses the injected primary flow on the inner curved surface and entrains the secondary flow. The basic idea behind the particle removal model is to take advantage of the free stream independence of the model in the outer part of the boundary layer and to retain the robust and accurate formulation Coanda flow formulation model in the near wall region. The discussion of this study proposes an investigation on the influence of various geometric parameters, pressure ratios and velocities towards the application of Coanda-effect for particle removal process. In order to achieve this, the $k-\varepsilon$ model is transformed into a $k-\omega$ formulation by means of a function that has the value one in the near wall region and zero away from the surface. Several numerical models of axisymmetric nozzles and guider for Coanda-effect have been built using the CFD software - Fluent with a preprocessor software - Gambit in order to obtain various results which then can be compared. In spurring the particle out of the surface, a high ratio of the induced mass flow rate is provided into the primary mass flow rate.

\section{A. Basic Design for Particle Removal Apparatus}

In specifying the design, some details must be examined, like attention on commonly used materials for camera lenses and covers can either be glass or polymer, which kinds of materials are easily exposed to dirt and other particles which cause poor visibility and etc. In the numerical simulations, a design of particle removal with Coanda-effect diffuser is created with the contraction efficient $k=2$. Fig. 3 shows basic designs of the proposed particle removal apparatus using Coanda-effect, with some adjustments. The fixed dimensions are $D_{1}=0.082 \mathrm{~m}, D_{2}=$ $0.08 \mathrm{~m}, D_{3}=0.01 \mathrm{~m}, L_{1}=0.08 \mathrm{~m}, L_{2}=0.047 \mathrm{~m}$ and $L_{3}=$ $0.033 \mathrm{~m}$, respectively (see Fig. 4). And also the recirculation zones that occur at the corners of the channel and beyond the contraction section have the lengths $S_{1}$ and $\mathrm{S}_{2}$, respectively.

\section{B Simulation Method}

The given parameters of the two-dimensional particle removal prototype for the numerical simulations are described in Table 1. In Numerical analysis, the code of Fluent is applied, in which the equations of continuity, time dependent full Navier-Stokes, energy, state and so on are used as the governing equation, and also the standard $k$ - $\varepsilon$ turbulence model to solve the turbulence stress criterion is utilized. By performing a computational study, the effect of various geometric parameters on the Coanda-effect particle removal prototype can be analysed especially for the turbulence analysis. The results in the distribution grid and meshing of the geometry which entrained for the Coanda particle removal prototype alongside the resulting solution 
with the variations of vector and contour for velocity and pressure distribution are shown in Fig. 5-7 while in accordance with similar application, respectively. The relative error between the simulated values $\mathrm{S}_{1}$ and $\mathrm{S}_{2}$ (whole channel, right and left corner) and the calculation domain obtained from the half-channel simulations is under $2 \%$.
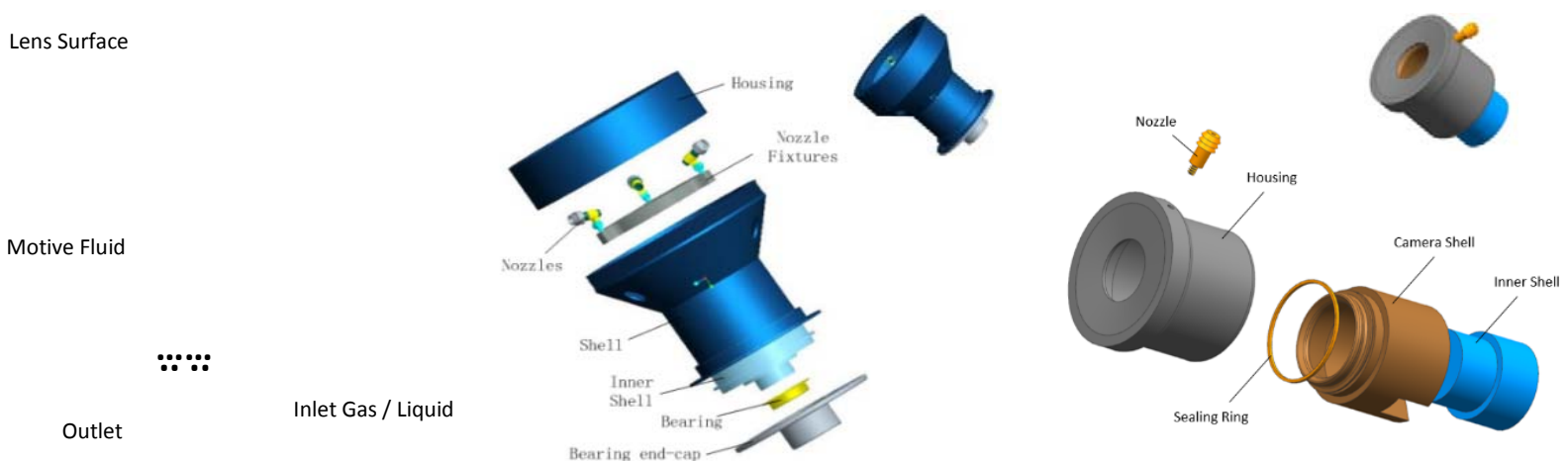

Figure 3. Design of particle removal system using Coanda-effect, Basic configuration (left), first prototype (middle), second prototype (right)

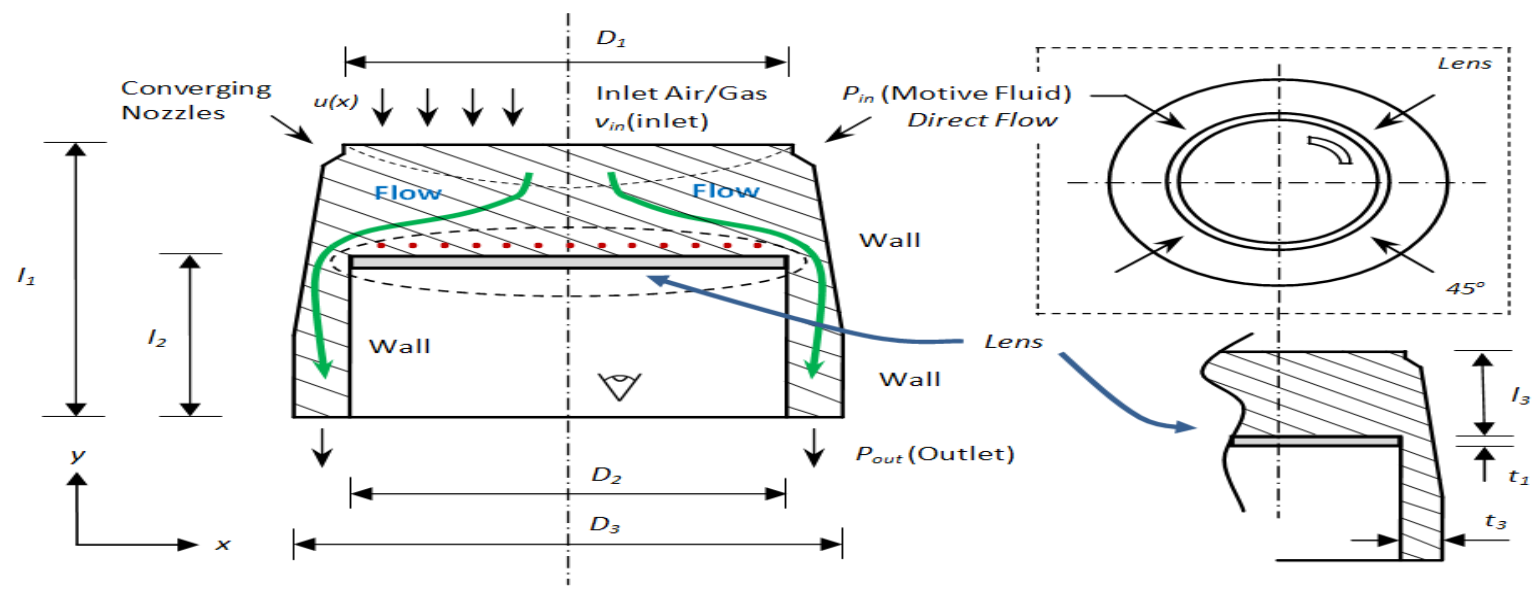

Figure 4. The geometrical model and the reference lengths of the Coanda-effect particle removal second prototype model with re-circulating zones character

TABLE I. COMPUTATIONAL SETTINGS FOR THE PARTICLE REMOVAL MODEL

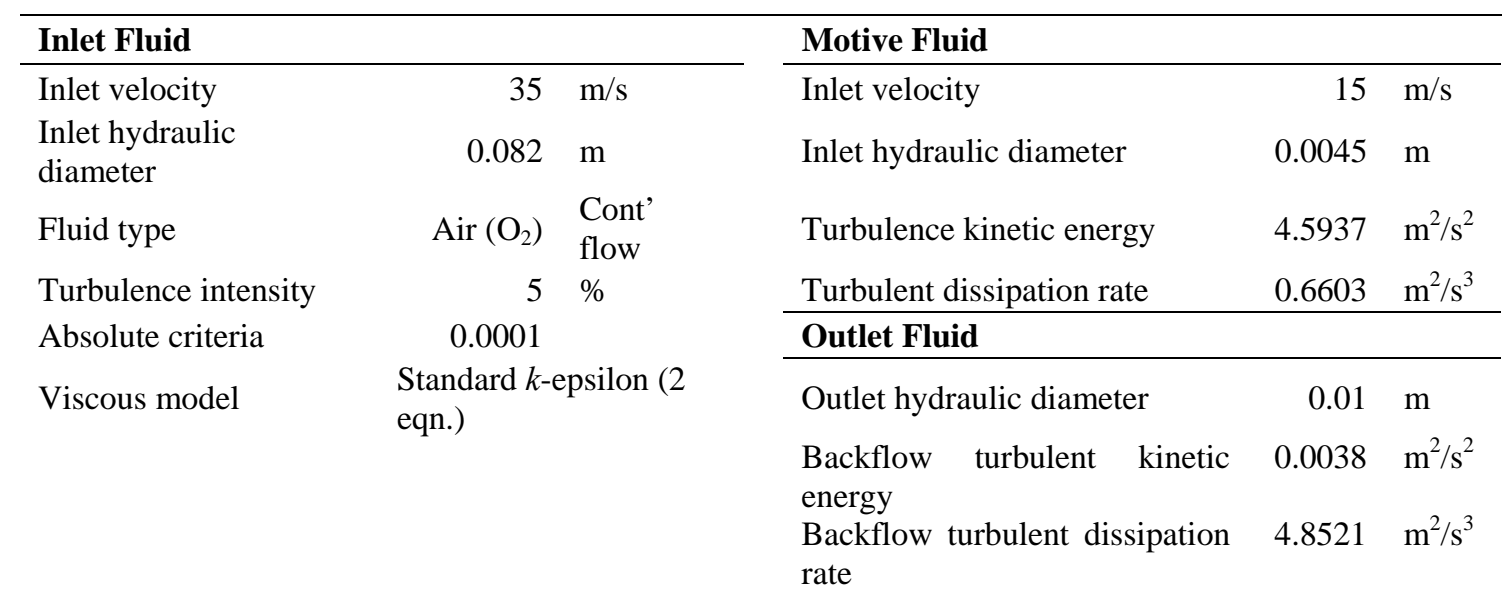



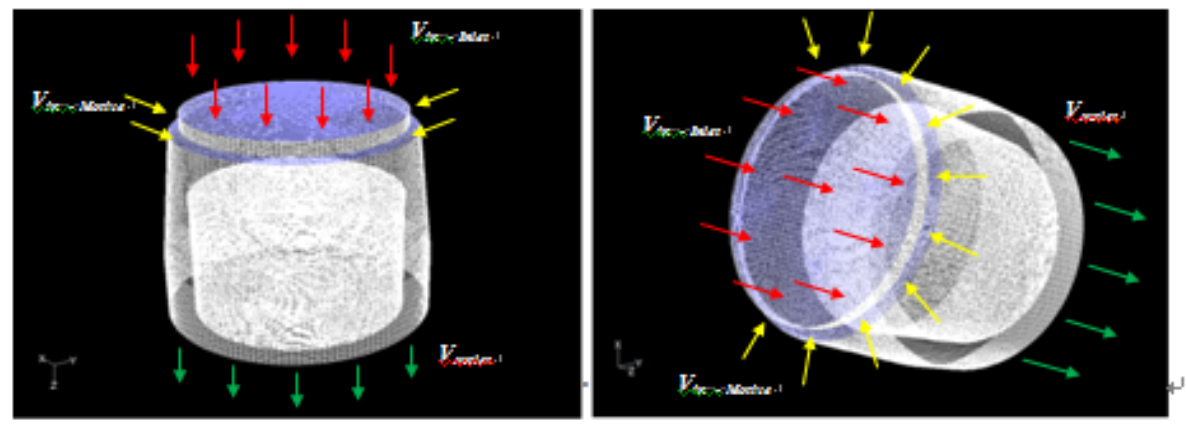

4

Figure 5. Coanda-effect particle removal prototype in 3-dimensional distribution meshing model

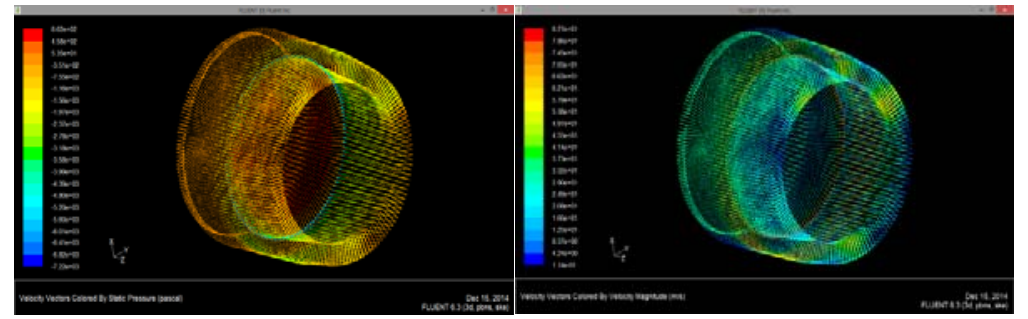

Figure 6. Flow regime on vector solution in full 3-dimensional channel model for $k=2$ and $\mathrm{Re}=3600$;

(a) Vector of velocity, (b) Vector of pressure with asymmetric orthogonal mesh
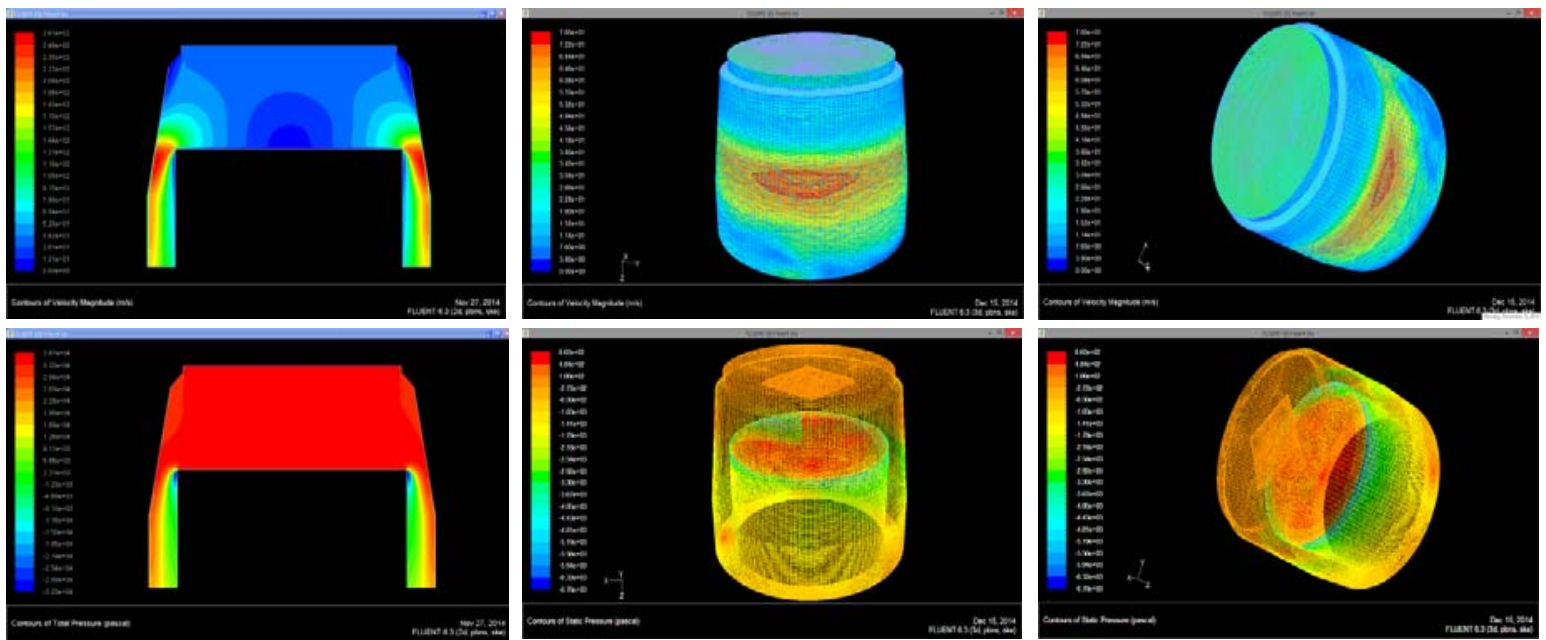

Figure 7. Flow regime on (top) contour of velocity and (bottom) contour of pressure solution in full 2 and 3-dimensional channel model for $k=2$ and Re $=$ 3600 with asymmetric orthogonal mesh

Generally, the camera lens cleaner parameters considered in the numerical simulation are based on the existing design parameters of a surface particle removal in our laboratory as other designs found in the literature. Similarly, the parameters considered are based on the characteristics of the selected condition and available related information in the literature. The results of numerical simulation of cleaning performance in terms of particle drag forces are presented in Table. 2, hence the simulation results explain that the residual for the prototype model shows linearity in most parameters, given in Fig. 8. 
TABLE II. Results of numerical analysis of particle drag force coefficient

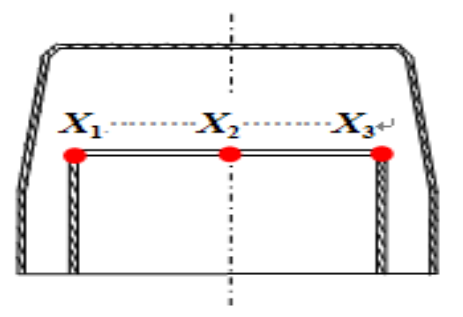

\begin{tabular}{cccccc}
\hline \multirow{2}{*}{ Point } & \multicolumn{2}{c}{ Force-(n) } & \multirow{2}{c}{ Coefficient-(n) } \\
& Pressure & Total & Pressure & Total \\
\hline$X_{1}$ & -2576 & -2566 & -4189 & -4189 \\
$X_{2}$ & -1652 & -1652 & -2698 & -2698 \\
$X_{3}$ & -1074 & -1074 & -1754 & -1754 \\
\hline
\end{tabular}

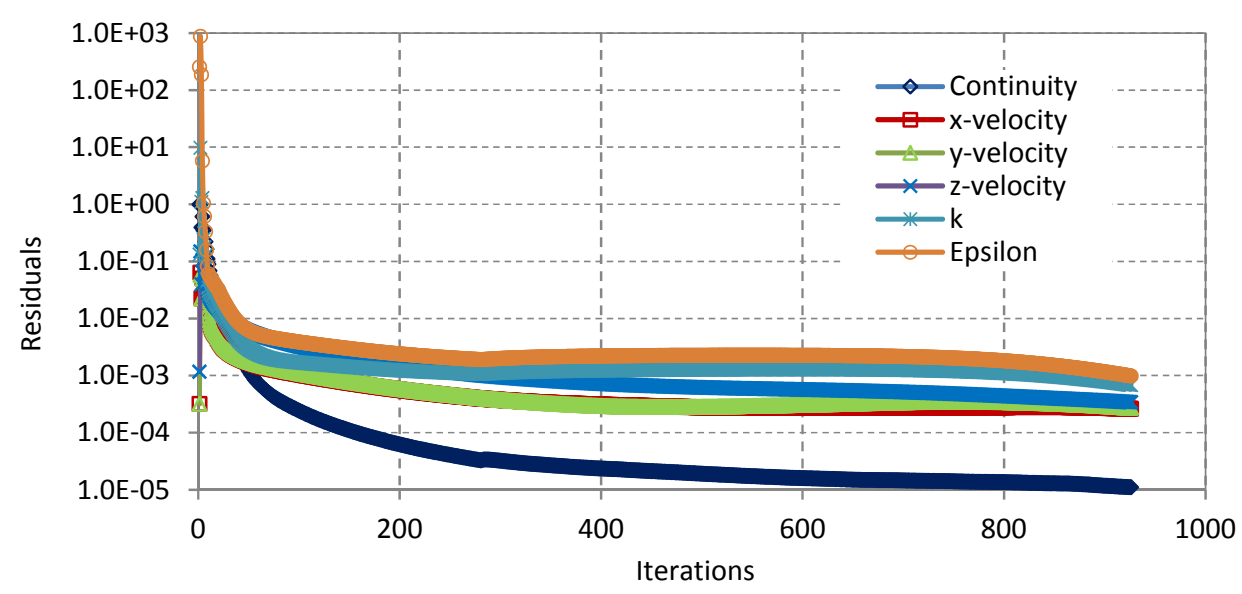

Figure 8. Scaled residual plot of the prototype model with various geometric parameters

\section{OPTIMIZATION PROBLEM FORMULATION}

Basic Concept and Application of Optimization to Particle Removal Performance. An optimization problem is one requiring the determination of the optimal (max. or min.) value of a given function, called the objective or fitness function, subject to certain defined restrictions, or constraints placed on the variables concerned [11]. Optimization could also be viewed as the process of making a decision or choosing the best out of available resources to achieve the most desired results. In optimization problems, we are interested in minimizing undesirable effects and/or maximizing desirable effects. Any of both of these will form the objective of optimization from which the objective function is formulated. An optimization problem could be single objective or multiobjective optimization. A general multi-objective minimization problem can be defined as follows:

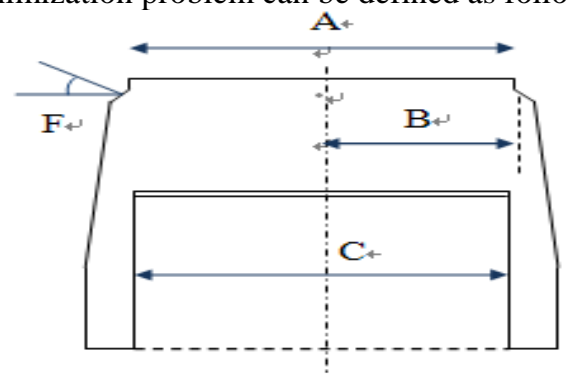

$$
\begin{gathered}
\min _{x \in X^{n}} f_{i}(x)=\left\{f_{1}(x), f_{2}(x), \ldots, f_{M}(x)\right\} \\
g(x) \geq 0, \quad h(x)=0
\end{gathered}
$$

Where $x$ is the vector of decision variables bounded by the decision space, $\boldsymbol{X}^{n_{x}}$ and $\mathrm{f}$ is the set of objectives to be minimized. The functions $g$ and $h$ are sets of inequality and equality constarints that define the feasible region of the $n_{x}$-dimensional continuous or discrete feasible solution space, respectively. Interface parameters for modeling and predicting pressure force and cleaning performance of coanda-effect particle removal apparatus in various condition described in Fig. 9.

Figure 9. Design of experiments for camera lens cover apparatus 
In a similar way to the optimization method discussed above, Fig. 10 shows the Pareto front or set of nondominated solutions for geometrical model and particle drag force coefficient, and the numerical values are presented in Table 3. The major difference between the first simulation and this second one is the range of indirect flow, according to the relevant decoupled model applied.
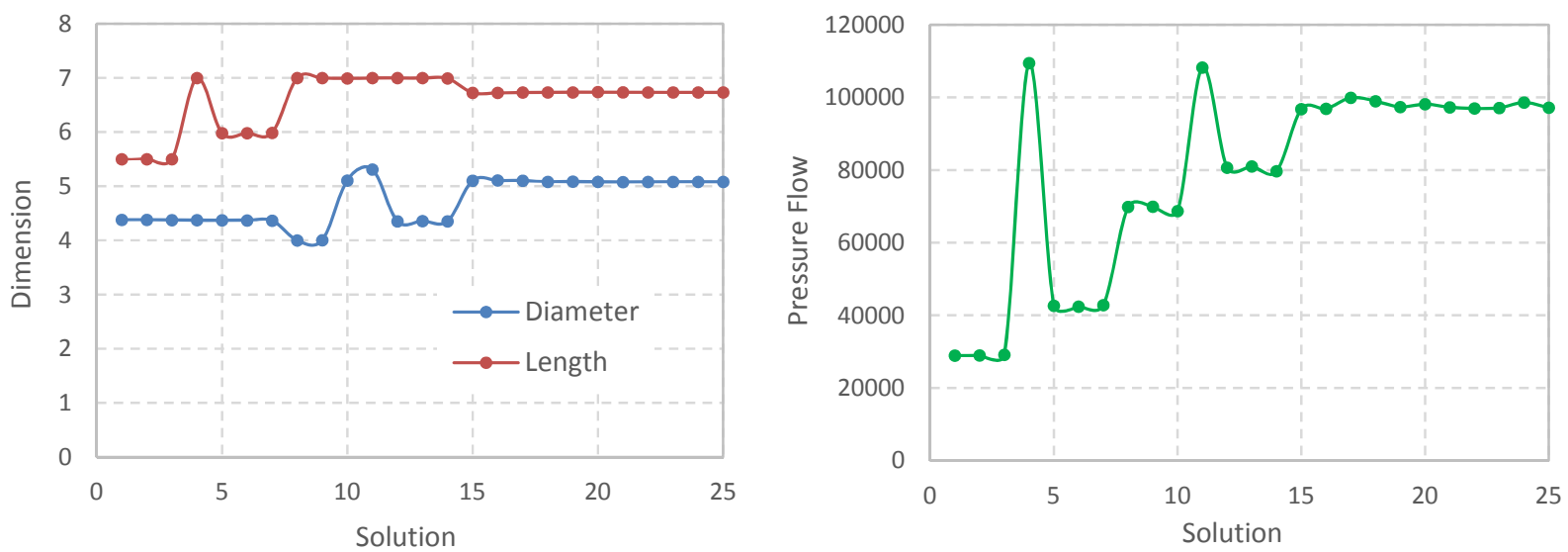

Figure 10. Optimization result for geometrical parameter (left) and pressure flow (right)

TABLE III. NUMERICAL ANALYSIS RESULTS OF PARTICLE DRAG FORCE COEFFICIENT

\begin{tabular}{c|c|c|c}
\hline $\begin{array}{c}\text { Solution } \\
\text { No. }\end{array}$ & $\begin{array}{c}\text { Radius, } r \\
(\mathrm{~cm})\end{array}$ & $\begin{array}{c}\text { Length, } l \\
(\mathrm{~cm})\end{array}$ & $\begin{array}{c}\text { Pressure, } P \\
(\mathrm{n})\end{array}$ \\
\hline 1 & 2.19 & 5.5 & 28940 \\
2 & 2.19 & 5.5 & 28981 \\
3 & 2.1875 & 5.5005 & 29157 \\
4 & 2.1875 & 7 & 109440 \\
5 & 2.186 & 5.981 & 42657 \\
& & & \\
25 & 2.54075 & 6.7344 & 97176 \\
\hline
\end{tabular}

\section{DISCUSSION AND CONCLUDING REMARKS}

This paper presents a numerical analysis and optimization of particle drag force analysis based on fluid flow and interface parameters in modeling. The following conclusion are made from the study:

- Application of Coanda-effect to the study of particle removal is generally a new area of research. This paper proposes a method for the application of innovative design on particle removal which has considered more adjustments on fluid flow variables with optimization for achieving optimized design, cleaning performances and particles drag forces coefficient.

- The validity of the results is limited to the cases given, since the described model differs in tangential component and momentum. Nonetheless, the regimes where the fluid assumption is not valid are usually some of the most baffling behaviors of fluid flow took place instead.
- The characteristic of Coanda flow with various parameters in different surfaces, involving smooth curved surfaces and a polygonal curved surface have been investigated. Using the Gambit and FLUENT Post-processor code, the distribution of pressure and the considered surfaces were analyzed particularly with the adaptation for Coanda-effect on particle removal application and optimization process done using i-SIGHT.

- Camera lens cover parameters, such as lens surface radius, camera cover length, and angle of attack are important design parameters having significant impact on cleaning performances of lens surfaces in various condition.

\section{ACKNOWLEDGEMENTS}

National Natural Science Foundation of China under Grant 60775060, Specialized Research Fund for the Doctoral Program of Higher Education under Grant 20122304110014, 20122304110014 and Harbin Science and Technology Innovation Talents Special Fund under Grant 2012RFXXG059.

\section{REFERENCES}

[1] McCormick and W. Barnies, Arodynamics, Aeronautics and Flight Mechanics. New York: John Wiley \& Sons, Inc. , 1979.

[2] B.B. Putra, X. He, and L. Z. Jie, "Study on the Design of Particle Removal System for Autonomous Robotic Vehicle," Advanced Materials Research Journal, vol. 694-697, pp. 1646-1651, 2013.

[3] J. D. Watson, "Thermal Spray Removal with Ultrahigh-Velocity Waterjets.," in Proceedings of 7th American Waterjet Conference, Seatle, WA, 1993, pp. 583-598.

[4] A. A. Busnaina and H. Lin, "Physical Removal of Nano-scale Defects from Surfaces in Advanced Semiconductor Manufacturing " presented at the IEEE/SEMI Conference and Workshop, 2002. 
[5] S. Keiichi and S. Yasuhiro, "Unstable Cavitation Behaviour in a Circular-Cylindrical Orifice Flow," Trans JSME, International Journal, Ser.B, vol. 45, pp. 638-645, 2002.

[6] R. Gouk, J. Papanu, and F. Li, "Tunable Droplet Momentum and Cavitation Process for Damage-free Cleaning of Chalenging Particles," presented at the Photomask Technology, 2008.

[7] A. Guha, R. M. Barron, and R. Balachandar, "An Experimental and Numerical Study of Water Jet Cleaning Process," Journal of Materials Processing Technology, vol. 211, pp. 610-618, 2011.
[8] R. Kobayashi, "On Development of Water Jet Jet Cutting Technology," presented at the 5th Symposium on Cavitation, 1987.

[9] A. F. Conn and M. T. Gracey, "Using Water Jets to Wash Out Explosives and Propellants," presented at the 9th Symp. on Jet Cutting Technlogy, 1988.

[10] S. J. Leach, G. L. Walker, and A. V. Smith, "Some Aspects of Rock Cutting by High Speed Water Jets," Philosophical Transactions of the Royal Society of London, vol. 260, pp. 295-310, 1966.

[11] K. A. Stroud, Advanced Engineering Mathematics. Houndmills: Palgrave MacMillan,
2003. 